Kong. Res. J. 7(1): 118-122, 2020

Publisher: Kongunadu Arts and Science College, Coimbatore.
ISSN 2349-2694, All Rights Reserved, https://www.krjournal.com

\title{
RESEARCH ARTICLE ON MODERATE NANO SOFT TOPOLOGICAL SPACE Priyalatha, S.P.R.*
}

Department of Mathematics, Kongunadu Arts and Science College,Coimbatore-641029, Tamil Nadu, India.

ABSTRACT: This paper study an alternative development of the nano soft topology which is known as moderate nano soft topology. Also, we define the characterization and properties are studied. Further we investigate moderate nano soft interior and moderate nano closure are discussed in details.

Keywords: Moderate nano soft topology, Moderate nano soft interior, Moderate nano soft closure, Moderate nano soft open .

\section{AMS Subject Classifications: 54A05 1. INTRODUCTION}

The theory of soft set was developed by Molodtsov, D. in 1999 [1]. It is a mathematical tool for handling problems involving imprecise, indeterminacy and inconsistent data, which was free from the difficulties that have troubled the usual theoretical approaches. Maji et al., [2] first applied soft set to solve the decision making problem. Shabir and Naz first introduced the notion of soft topological spaces, which are defined over an initial universe with a fixed set of parameters, and showed that a soft topological space gives a parameterized family of topological spaces Shabir, M., and Naz, M.[3] . The concept of nano topology was introduced by Lellis Thivagaret al. in the year 2013 [4]. Several authors [5-11] have contributed in soft set and nano topological spaces. The motive of this paper is to study about the modification of nano soft nano topology is called as moderate nano soft topology. Likewise, we study the MS- approximation space and also, we define their properties of MS-lower, MS- upper approximations and MS- boundary region. We discuss their properties and examples are defined.

\section{Preliminary}

In this section we recall some relevant definitions.

Definition 2.1 [4]: Let $U$ be a non-empty finite set of objects called the universe and $\mathrm{R}$ be an equivalence relation on $U$ named as the indiscernibility relation. Elements belonging to the same equivalence class are said to be indiscernible with one another. The pair $(U$, $R$ ) is said to be the approximation space. Let $X \subseteq U$.

(i) The lower approximation of $\mathrm{X}$ with respect to $\mathrm{R}$ is the set of all objects, which can be for certain classified as $\mathrm{X}$ with respect to $\mathrm{R}$ and it is denoted by $L_{R}(X)$. That is, $L_{R}(X)=$ $\bigcup_{x \in U}\{R(x): R(x) \subseteq X\}$, where $\mathrm{R}(\mathrm{x})$ denotes the equivalence determined by $\mathrm{x}$.

(ii) The upper approximation of $\mathrm{X}$ with respect to $\mathrm{R}$ is the set of all objects, which can be possibly classified as $\mathrm{X}$ with respect to $\mathrm{R}$ and it is denoted by $U_{R}(X)$. That is, $U_{R}(X)=$ $\cup_{x \in U}\{R(x): R(x) \cap X \neq \phi\}$, where $\mathrm{R}(\mathrm{x})$ denotes the equivalence determined by $\mathrm{x}$.

(iii) The boundary region of $X$ with respect to $R$ is the set of all objects, which can be classified neither as $\mathrm{X}$ nor as not $-\mathrm{X}$ with respect to $\mathrm{R}$ and it is denoted by $B_{R}(X)$. That is,

$$
B_{R}(X)=U_{R}(X)-L_{R}(X) .
$$

Definition 2.2 [4]: Let $U$ be an universe, $\mathrm{R}$ be an equivalence relation on $U$ and $\tau_{R}(X)=\left\{U, \phi, L_{R}(X)\right.$, $\left.\mathrm{U}_{\mathrm{R}}(\mathrm{X}), \mathrm{B}_{\mathrm{R}}(\mathrm{X})\right\}$ where $\mathrm{X} \subseteq \mathrm{U}, \tau_{\mathrm{R}}(\mathrm{X})$ satisfies the following axioms:

(i) $\quad U$ and $\phi \in \tau_{\mathrm{R}}(\mathrm{X})$.

(ii) The union of the elements of any subcollection of $\tau_{R}(X)$ is in $\tau_{R}(X)$.

(iii) The intersection of the elements of any finite sub-collection of $\tau_{R}(X)$ is in $\tau_{R}(X)$.

That is, $\tau_{R}(X)$ forms a topology on $U$ called the nano topology on $U$ with respect to $X$. We call $\left(U, \tau_{R}(X)\right)$ as the nano topological space. The elements of $\tau_{R}(X)$ are called nano-open sets.

Definition 2.3: $[3,11]$ A soft set $(F, A)$ denoted by $F_{A}$ on the universe $U$ is defined by the set of ordered pairs $\mathrm{F}_{\mathrm{A}}=\{(\mathrm{e}, \mathrm{F}(\mathrm{e})): \mathrm{e} \in \mathrm{E}, \mathrm{F}(\mathrm{e}) \in \mathrm{P}(\mathrm{U})\}$, where $\mathrm{F}: \mathrm{E} \rightarrow \mathrm{P}(U)$ such that $\mathrm{F}(\mathrm{e})=\phi$ if $\mathrm{e} \in \mathrm{A}$. Here, $\mathrm{F}$ is called an approximate function of the soft set $F_{A}$. The set $F(e)$ is

${ }^{*}$ Correspondence: Priyalatha, S.P.R., Department of Mathematics, Kongunadu Arts and Science College,Coimbatore, Tamil Nadu, India.E-mail: spr.priyalatha@gmaill.com 
called e-approximate value set or e-approximate set which consists of related objects of the parameter $e \epsilon$ E.

Definition 2.4: $[3,6,11]$ A soft set $(F, A)$ over $U$ is said to be NULL soft set denoted by $\phi$, if $\forall \mathrm{e} \in \mathrm{A}, \mathrm{F}(\mathrm{e})=\phi$.

Definition 2.5:[3, 6, 11] A soft set $(F, A)$ over $U$ is said to be an absolute soft set denoted by ${ }^{\mathcal{U}} \mathrm{U}$, if $\forall \mathrm{e} \in \mathrm{A}$, $F(e)=U$.

Definition 2.6: $[3,6,11]$ A union of two soft sets $F_{A}$ and $G_{B}$ over a common universe $U$ is the soft set $(H, C)$,

where $C=A \cup B$, and $\forall e \in C$,

$H(e)=\left\{\begin{array}{l}F(e) \text { ife } \in A-B \\ G(e) \text { ife } \in B-A \\ F(e) \cup G(e) \text { ife } \in A \cap B\end{array}\right.$

We write $\mathrm{F}_{\mathrm{A}} \cup \mathrm{G}_{\mathrm{B}}=\mathrm{H}_{\mathrm{C}}$.

Definition 2.7:[ 3, 6, 11] An intersection of two soft sets $F_{A}$ and $G_{B}$ over a common universe $U$ is the soft set $\mathrm{H} c$, where $\mathrm{C}=\mathrm{A} \cap \mathrm{B}$, and $\forall \mathrm{e} \in \mathrm{C}, \mathrm{H}(\mathrm{e})=\mathrm{F}(\mathrm{e}) \cap \mathrm{G}(\mathrm{e})$. We write $F_{A} \cap G_{B}=H_{c}$.

Definition2.8:[3, 6, 11] For two soft sets $F_{A}$ and $G_{B}$ over a common universe $U$, we say that $F_{A}$ is a soft subset of $G$, $\mathrm{B}$ if (i) $A \subset B$ and (ii) $\forall e \in A, F(e)$ and $G(e)$ are identical approximations. we write $F_{A} \subset G_{B}$. $F_{A}$ is said to be a soft super set of $G_{B}$, if $G_{B}$ is a soft subset of $\mathrm{F}_{\mathrm{A}}$. We denote it by $\mathrm{F}_{\mathrm{A}} \supset \mathrm{G}_{\mathrm{B}}$.

\section{MODERATE ON NANO SOFT TOPOLOGY}

Inthissection, wedefinethenanotopologic alspaceinducedbysoftidealissaidtobe"nano soft ideal topological space" and their properties areinvestigated.

Definition 3.1: Let $U$ be nonempty finite universe, $F_{A}$ be a soft set over $\mathrm{U}$ where $\mathrm{F}$ is a map $F: A \rightarrow P(\mathrm{U})$.Let $\psi: \mathrm{U} \rightarrow P(\mathrm{U})$ be another map defined as $\psi(x)=\{a: x \in F(a)\}$. Then the pair $(\mathrm{U}, \psi)$ is a MS-approximation space and for any $X \subseteq \mathrm{U}$.

(i) The MS-lower approximation is defined as $L_{\psi}(X)=\{x \in X: \psi(x)=\psi(y)$ for all $y$ $\in X c\}$, where $X^{c}=\mathrm{U}-X$

(ii) TheMSupperapproximationisdefinedas $U_{\psi}(X)=\{X$ $\in X: \psi(x)=\psi(y)$ forsome $y \in X\}$.

(iii) TheMSboundaryregionisdefinedas $B_{\psi}(X)=U_{\psi}(X)$ $-L_{\psi}(X)$.
Definition 3.2: Let $U$ be a nonempty finite set of objects called the universe, $F_{A} \subseteq F_{E}$ is ansoftsetoverU.Then $(\mathrm{U}, \psi)$ isanorderedpairofMS -approximationspaceand $\tau_{\psi}^{*}(X)=\{\mathrm{U}$,

$\left.\left.\phi, L_{\psi}^{*}(X), U_{\psi}^{*}(X), B_{\psi}^{*}(X)\right\}\right)$, where $X \subseteq \mathrm{U}$.Thatis, $\tau_{\psi}^{*}(X) \mathrm{f}$ ormsamoderatenanosofttopologyonUhavingth eatmostfiveelementsofsoftsetandtripleordered pairof $\left(\mathrm{U}, \tau_{\psi}^{*} E\right)$ is

calledamoderatenanosofttopologicalspaceoverU withrespecttoX,thenthemembersof

$\tau_{\psi}^{*}(X)$ aresaidtobemoderatenanosoftopensetsi $\mathrm{nU}$.

Example 3.3: Let $\mathrm{U}=\{(a, b, c, d, e, f\}$ be the universe, $\quad \mathrm{E}=\quad\left\{\mathrm{e}_{1}, \mathrm{e}_{2}, \mathrm{e}_{3}, \mathrm{e}_{4}, \mathrm{e}_{5}\right\}$ and $A=\left\{e_{1}, e_{2}, e_{3}\right\}$ beasetofparameters. Let $X=\{a, c, f\}$ $\subseteq$ Usuchthat $\psi(a)=\left\{e_{1}, e_{2}\right\}, \psi(b)=\left\{e_{2}\right\}=\psi(c), \psi(d)$ $=\left\{e_{1}\right\}, \psi(e)=\left\{e_{3}\right\}, \psi(f)=\left\{e_{1}, e_{3}\right\}$.Since $c \in X$ and $b \in X^{c}$. Then $L_{\psi}(X)=$

$\{a, f\}, U_{\psi}(X)=\{a, b, c, f\}$, and $B_{\psi}(X)=\{b, c\}$.Hence $\tau_{\psi}(X)$ $=\{\mathrm{U}, \varphi,\{a, f\},\{a, b, c, f\},\{b, c\}\}$ is a moderate nano soft topologicalspace.

Definition 3.4: Let $F_{A} \subseteq F_{E}$ be a soft set over $\mathrm{U}$ and $(\mathrm{U}, \psi)$ be a MS- approximation space and $X \in U$. We define the characterization of five basic types of moderate nano soft topological space as followsas:

(i) $\operatorname{If} L_{\psi}(X)=\phi$ and $U_{\psi}(X)=\mathrm{U}$, then $\tau_{\psi}(X)=\{\mathrm{U}$, $\phi \quad\}$ iscalledas MNS-indiscretetopology onU.

(ii) If $L_{\psi}(X)=U_{\psi}(X)=\mathrm{U}$, thentheMNStopology, $\tau_{\psi}(X)=\left\{\mathrm{U}, \phi, L_{\psi}(X)\right\}$.

(iii) If $L_{\psi}(X)=\phi \quad$ and $U_{\psi}(X)=\mathrm{U}$, thenMNStopology, $\tau_{\psi}(X)=\left\{\mathrm{U}, \phi, U_{\psi}(X)\right\}$.

(iv) If $L_{\psi}(X)=\phi \quad$ and $U_{\psi}(X)=\mathrm{U}$, thenMNS-topology $\tau_{\psi}(X)=\left\{\mathrm{U}, \phi, L_{\psi}(X), B_{\psi}(X)\right\}$.

(v) If $\quad L_{\psi}(X)=$ $U_{\psi}(X)$, where $L_{\psi}(X)=\phi$ and $U_{\psi}(X)=\mathrm{U}$, thenMN S-discretetopologyon $\tau_{\psi}(X)=\left\{\mathrm{U}, \phi, L_{\psi}(X)\right.$, $\left.U_{\psi}(X), B_{\psi}(X)\right\}$.

Proposition 3.5: Let $F_{A}$ be a soft set over $U$ and $(\mathrm{U}, \psi)$ is an approximation space.Then

$$
L_{\psi}(X) \subseteq \mathrm{X} \subseteq U_{\psi}(X)
$$



(ii) $\quad L_{\psi}(\phi)=\phi$ and $U_{\psi}(\phi)=\phi$
(iii) $\quad L_{\psi}(U) \subseteq U \subseteq U_{\psi}(U)$
(iv) $\quad \mathrm{L}_{\psi}(\mathrm{X} \cap Y)=L_{\psi}(X) \cap L_{\psi}(Y)$
(v) $\quad U_{\psi}(X \cap Y) \subseteq U_{\psi}(X) \cap U_{\psi}(Y)$
(vi) $\quad L_{\psi}(X \cup Y) \supseteq L_{\psi}(X) \cup L_{\psi}(Y)$
(vii) $\quad U_{\psi}(X \cup Y)=U_{\psi}(X) \cup U_{\psi}(Y)$
(viii) $\quad X \subseteq Y \Rightarrow L_{\psi}(X) \subseteq L_{\psi}(Y), U_{\psi}(X) \subseteq U_{\psi}(Y)$
(ix) $\quad U_{\psi}(X)=\left[L_{\psi}\left(X^{C}\right)\right]^{C}$
(x) $\quad L_{\psi}(X)=\left[U_{\psi}\left(X^{C}\right)\right]^{C}$
(xi) $\quad U_{\psi}\left(L_{\psi}(X)\right)=\left[L_{\psi}(X]\right.$
(xii) $\quad L_{\psi}\left(L_{\psi}(X)\right)=\left[L_{\psi}(X]\right.$
(xiii) $\quad U_{\psi}\left(L_{\psi}(X)\right)=\left[U_{\psi}(X]\right.$
(xiv) $\quad U_{\psi}\left(U_{\psi}(X)\right)=\left[U_{\psi}(X]\right.$

Proof:

(i),(ii) and (iii) is directly from the definition.

(iv) Let $\mathrm{u} \in L_{\psi}(X) \cap L_{\psi}(Y)$. Then $\mathrm{u} \in L_{\psi}(X)$ and $\mathrm{u} \in L_{\psi}(Y)$.By definition, $\mathrm{u} \in X$, $\psi(u) \neq \psi(v) \forall v \in X^{C} \quad$ and $\quad \mathrm{u} \quad \in Y$ $\psi(u) \neq \psi(z) \forall z \in Y^{C}$ which implies that $\mathrm{u} \in X \cap Y$ $\psi(u) \neq \psi(v) \forall v \in X^{C} \cup Y^{C} \quad=$ $(X \cap Y)^{C} \Rightarrow u \in L_{\psi}(X \cap Y)$. Conversely, let $u \in L_{\psi}(X \cap Y) \quad, \quad$ u $\in X \cap Y, \quad \psi(u) \neq \psi(v)$ $\forall v \in(X \cap Y)^{C}$. Therefore we have $u \in X, u \in Y$, $\psi(u) \neq \psi(v) \quad \forall v \in X^{C} \cup Y^{C}$.Also, we have $L_{\psi}(X) \cap L_{\psi}(Y) \quad \forall v \in(X \cap Y)^{C}$.Therefore, we have $\mathrm{u} \in X, \psi(u) \neq \psi(v) \forall v \in X^{C}$ and $\mathrm{u} \in Y$ $\psi(u) \neq \psi(z) \forall z \in Y^{C}$,which implies that $\mathrm{u}$ $\in L_{\psi}(X)$ and $\mathrm{u} \in L_{\psi}(Y)$. Since $\mathrm{u} \in L_{\psi}(X \cap Y)$ .Hence $\mathrm{L} \psi(\mathrm{X} \cap Y)=L_{\psi}(X) \cap L_{\psi}(Y)$. (v) Let $\mathrm{u} \in U_{\psi}(X \cup Y)$. We have $\psi(u)=\psi(v)$ for some $\mathrm{v} \in X \cap Y$.Therefore we can $\psi(u)=\psi(v)$ for some $\mathrm{v} \in X$ and $\psi(u)=\psi(v)$ for some $\mathrm{v} \in Y$. Since $\mathrm{u} \in U_{\psi}(X)$ and $\mathrm{u} \in U_{\psi}(Y)$. That is $\mathrm{u} \in L_{\psi}(X)$ $\cap L_{\psi}(Y)$.Hence $U_{\psi}(X \cap Y) \subseteq U_{\psi}(X) \cap U_{\psi}(Y)$.

(vi) Let $\mathrm{u} \in L_{\psi}(X) \cup L_{\psi}(Y)$. We have $\mathrm{u} \in L_{\psi}(X)$ or $\mathrm{u} \in L_{\psi}(Y)$.Since $\mathrm{u} \in X, \psi(u) \neq \psi(v) \forall v \in X^{C}$ or $\mathrm{u}$ $\in Y, \psi(u) \neq \psi(z) \forall z \in Y^{C}$.Therefore, in particular $\mathrm{u} \in X \cup Y, \psi(u) \neq \psi(v) \forall v \in(X \cup Y)^{C}$. Since $X \subseteq X \cup Y \Rightarrow(X \cup Y)^{C} \subseteq X^{C}, \mathrm{u}$ $\Rightarrow x \in L_{\psi}(X \cup Y)$. Hence $L_{\psi}(X \cup Y) \supseteq L_{\psi}(X) \cup L_{\psi}(Y)$

(vii) Let $\mathrm{u} \in U_{\psi}(X) \cup U_{\psi}(Y)$.We have $\mathrm{u}$ $\in U_{\psi}(X)$ or $\mathrm{u} \in U_{\psi}(Y)$, which implies that $\psi(u)=\psi(v) \forall v \in X$ or $\psi(u)=\psi(z) \forall z \in Y$.

Therefore, we have $\psi(u)=\psi(v) \forall v \in X \cup Y$.Thus, $\mathrm{u} \in U_{\psi}(X \cup Y)$.Hence $U_{\psi}(X \cup Y) \supseteq U_{\psi}(X) \cup U_{\psi}(Y)$.Conversely, let u $\in U_{\psi}(X \cup Y)$ in particular $\mathrm{u} \in X \cup Y$, $\psi(u)=\psi(v)$ for some $v \in X \cup Y$. Since $\mathrm{u} \in X$, $\psi(u)=\psi(v) \forall v \in X$ or $\mathrm{u} \in Y$, $\psi(u)=\psi(y) \forall y \in Y$. Both the above conditions imply thatu $\in U_{\psi}(X)$ or $\mathrm{u} \in U_{\psi}(Y)$.Thus, $\mathrm{u}$ $\in U_{\psi}(X \cup Y)$ and so $U_{\psi}(X \cup Y) \subseteq U_{\psi}(X) \cup U_{\psi}(Y)$.Hence $U_{\psi}(X \cup Y)=U_{\psi}(X) \cup U_{\psi}(Y)$.

(viii) Let $X \subseteq Y$ and $\mathrm{u} \in L_{\psi}(X)$, which implies that $\psi(u) \neq \psi(v) \quad \forall v \in X^{C} \quad$. As $Y^{C} \subseteq X^{C}$, $\psi(u) \neq \psi(z) \forall z \in Y^{C}$. Thus, $\mathrm{u} \in L_{\psi}(Y)$.We get $L_{\psi}(X) \subseteq L_{\psi}(Y)$. Now let $v \in L_{\psi}(X)$, that is $\psi(v) \neq \psi(z) \quad \forall v \in X \quad$.Since $\quad X \subseteq Y$, $\psi(v)=\psi(z) \forall z \in Y$. Thus, $v \in L_{\psi}(Y)$.We get $U_{\psi}(X) \subseteq U_{\psi}(Y)$. 
(ix) Let $\mathrm{u} \in\left[L_{\psi}\left(X^{C}\right)\right]^{C}=U-L_{\psi}\left(X^{C}\right)$.Then $\mathrm{u} \in U$ $\mathrm{u} \notin L_{\psi}\left(X^{C}\right)$.This gives that $\psi(u)=\psi(v)$ for some $v \in\left(X^{C}\right)^{C}=X$, thus, $\mathrm{u} \in U, \psi(u)=\psi(v)$ for some $v \in X$, and so $\mathrm{u} \in U_{\psi}(X)$. Conversely, let $\mathrm{u}$ $\in U_{\psi}(X)$.Then, $\mathrm{u} \in U, \psi(u)=\psi(v)$ for some $v \in X$, So that $\mathrm{u} \notin L_{\psi}\left(X^{C}\right)$ that is $\mathrm{u} \in U-L_{\psi}\left(X^{C}\right)$ .Thus $\mathrm{u} \quad \in\left[L_{\psi}\left(X^{C}\right)\right]^{C}$. Hence $U_{\psi}(X)=\left[L_{\psi}\left(X^{C}\right)\right]^{C}$.

(x) Let $\mathrm{u} \in\left[U_{\psi}\left(X^{C}\right)\right]^{C}=U-U_{\psi}\left(X^{C}\right)$.Then $\mathrm{u} \in U$ , $\mathrm{u} \notin U_{\psi}\left(X^{C}\right)$.By definition $\psi(u) \neq \psi(v)$ for all $v \in X^{C}$.This shows that $\mathrm{u} \in X$ because otherwise, if $\mathrm{u} \in U-X, \psi(u)=\psi(v)$ which contradicts the above condition .Thus $\mathrm{u} \in X, \psi(u) \neq \psi(v)$ for all $v \in X^{C}$, and so $\mathrm{u} \in L_{\psi}(X)$. Conversely, let $\mathrm{u}$ $\in L_{\psi}(X)$.Then, by definition $\mathrm{u} \in X, \psi(u) \neq \psi(v)$ for all $v \in X^{C}$, which gives $\mathrm{u} \notin U_{\psi}\left(X^{C}\right)$ that is, $\mathrm{u}$ $\in U-U_{\psi}\left(X^{C}\right)$.This implies that $\mathrm{u} \in\left[U_{\psi}\left(X^{C}\right)\right]^{C}$. Hence $L_{\psi}(X)=\left[U_{\psi}\left(X^{C}\right)\right]^{C}$.

(xi) From (i) we get $L_{\psi}(X) \subseteq U_{\psi}\left(L_{\psi}(X)\right)$ .Consider $u \subseteq U_{\psi}\left(L_{\psi}(X)\right)$. Then we have $\mathrm{u} \in U$ $\psi(u)=\psi(v)$ for some $v \in L_{\psi}(X)$. By definition 3.1 we have $v \in X, \psi(v) \neq \psi(z) \forall z \in X^{C}$. As $\psi(u)=\psi(v)$, we get $\psi(u) \neq \psi(z) \forall z \in X^{C}$. Thus, $\mathrm{u} \notin X^{C}$ that is, $\mathrm{u} \in X$ and so get $u \in L_{\psi}(X)$ $L_{\psi}(X) \subseteq L_{\psi}(Y)$. Hence $L_{\psi}(X) \supseteq U_{\psi}\left(L_{\psi}(X)\right)$ and now we conclude that $L_{\psi}(X)=U_{\psi}\left(L_{\psi}(X)\right)$. (xii) From definition 3.1 we have $L_{\psi}\left(L_{\psi}(X)\right)=\left\{u \in L_{\psi}(X): \psi(x) \neq \psi(y)\right.$ for all $\left.v \in\left(L_{\psi}(X)\right)^{C}\right\}$. By (i) we have $L_{\psi}(X) \subseteq X$ which gives $L_{\psi}(X)^{C} \supseteq X^{C}$.Thus in particular we have $L_{\psi}\left(L_{\psi}(X)\right)=\left\{u \in L_{\psi}(X): \psi(x) \neq \psi(y)\right.$ for all $\left.v \in\left(L_{\psi}(X)\right)^{C}\right\}=L_{\psi}(X)$. (xiii) By (i) we have $U_{\psi}\left(L_{\psi}(X)\right) \subseteq U_{\psi}(X)$. Conversely, if $v \notin U_{\psi}\left(L_{\psi}(X)\right)$, then by definition 3.1, either $\quad v \notin U_{\psi}(X) \quad$ or $\psi(v) \neq \psi(z) \forall z \in\left[U_{\psi}(X)\right]^{C}$. If $v \notin U_{\psi}(X)$ then we get our required result .In the later case $\psi(v) \neq \psi(z)$ for some $z \in\left[U_{\psi}(X)\right]^{C}$ so that $z \notin U_{\psi}(X)$ that is $\psi(z) \neq \psi(w)$ for all $w \in X$. But then, $\psi(v)=\psi(z), \psi(v)=\psi(w)$, for all $w \in X$. (i).Therefore, we have $v \in\left[L_{\psi}(X)\right]^{C}$ that is $v \in U_{\psi}\left(L_{\psi}(X)\right)=U_{\psi}(X) \quad$,by

$U_{\psi}(X) \subseteq U_{\psi}\left(L_{\psi}(X)\right)$ .Hence $U_{\psi}(X)=U_{\psi}\left(L_{\psi}(X)\right)$.

(xiv) By (i) $U_{\psi}\left(U_{\psi}(X)\right) \supseteq U_{\psi}(X)$. For the reverse inclusion, let $u \in U_{\psi}\left(U_{\psi}(X)\right)$, then by definition 3.1, $\psi(u)=\psi(v)$ for some $v \in U_{\psi}(X)$ that is $\psi(v)=\psi(z)$ for some $z \in X$.This implies that $\psi(u)=\psi(z)$ for some $z \in X$.So, $u \in U_{\psi}(X)$.Thus $U_{\psi}\left(U_{\psi}(X)\right) \subseteq U_{\psi}(X)$ .Hence $U_{\psi}(X)=U_{\psi}\left(U_{\psi}(X)\right)$.

Proposition 3.6: Let $\left(U, \tau_{\psi}^{*} E\right)$ be moderate nano soft topological space and $\mathrm{F}_{\mathrm{A}}$ be a soft set over $\mathrm{U}$ and $(\mathrm{U}, \psi)$ is an approximation space. Then for any $\mathrm{X} \in P(U)$, either both $u, v \in U_{\psi}(X)$ or $u, v \notin U_{\psi}(X)$

Proof: If $u \in U_{\psi}(X)$ then $\psi(u)=\psi(v)$ for some $z \in X$.But, as, $\psi(u)=\psi(v)$,so $\psi(v)=\psi(z)$ for some $z \in X$.This implies that $v \in U_{\psi}(X)$.

Definition 3.7: Let $\left(U, \tau{ }_{\psi}^{*} E\right)$ be a moderate soft nano topological space over $U$. The moderate nano soft closure of $F_{N}{ }^{\prime}$ is the soft set. That is $\mathrm{M}_{s} c l\left(F_{N}{ }^{\prime}\right)=$ $\cap\left\{G_{n}{ }^{\prime}: G_{n}{ }^{\prime}\right.$ is moderate nano soft closed and $\left.F_{n}{ }^{\prime} \subseteq G_{n}{ }^{\prime}\right\}$. Clearly $\mathrm{M}_{s} c l\left(F_{N}{ }^{\prime}\right)$ is the smallest moderate nano soft closed set that containing $F_{N}$ '.

Definition 3.8: Let $\left(\mathrm{U}, \tau_{\psi}^{*} E\right)$ be a moderate soft nano topological space over $U$. The moderate nano soft interior of $F_{N}{ }^{\prime}$ is the soft set. That is $\mathrm{M}_{\operatorname{sint}}\left(F_{N}{ }^{\prime}\right)=$ 
$\cup\left\{O_{n}{ }^{\prime}: O_{n}{ }^{\prime}\right.$ is moderate nano soft open and $\left.O^{\prime}{ }_{n} \subseteq F^{\prime}{ }_{n}\right\}$. Clearly $\mathrm{M}_{\text {sint }}\left(F_{N}{ }^{\prime}\right)$ is the largest

\section{REFERENCES}

1. Molodtsov, D. (1999). Soft set theory-first results, Computers and Mathematics with Applications 37:19-31.

2. Maji, P.K., Biswas, R. and Roy, A.R. (2001). Fuzzy soft set theory, Journal of Fuzzy Mathematics.3:589-602.

3. Shabir, M. and Naz, M. (2011). On soft topological spaces, Computers and Mathematics with Aplication61:1786-1799.

4. Lellis Thivagar, M. and Carmel Richard, (2013). On nano forms of weakly open sets, International Journal of Mathematics and Statistics Invention 1(1):31-37

5. Aktas, H. and Cagman, N. (2007). Soft sets and soft groups. Information Science177: 27262735.

6. Bayramov,S. and Gunduz, C.(2018). A new approach to separability and compactness in soft topologicalspaces. TWMS Journal of Pure and Applied Mathematics 9:82-93. moderate nano soft open set that is contained in $F_{N}{ }^{\prime}$.

7. Cagman, N; Karatas, S. and Enginoglu, S. (2011). Soft topology. Computers and Mathematics withApplications62: 351-358.

8. Chen, D., Tsang, E.C.C., Yeung, D.S., Wang, X.,(2005).The parametrization reduction of soft sets and its applications, Computers and Mathematics with Appication49:757-763.

9. Feng, F., Jun, Y. B., Liu, X.Y. and Li, L.F.(2010).An adjustable approach to fuzzy soft set based decision making, Journal of Computational and Applied Mathematics234:10-20.

10. Lellis Thivagar, M. and Priyalatha, S.P.R.(2017). An innovative approach on nano soft topological space, South East Asian journal of Mathematics and Mathematical sciences13(2): 47-62.

11. Tozlu, N., and Yuksel, S. (2017). Soft A-sets and Soft B-sets in Soft Topological Spaces, Mathematical Sciences and Applications ENotes 5(2):17-25.

\section{About The License}

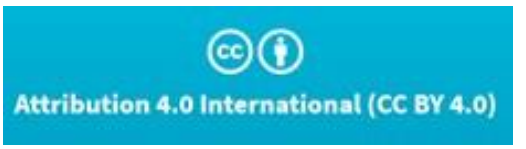

The text of this article is licensed under a Creative

Commons Attribution 4.0 International License 